\title{
Análise do efeito da radiofrequência no tratamento de flacidez cutânea relacionada ao processo de envelhecimento: revisão integrativa
}

\author{
Analysis of the effect of radiofrequency in the treatment of skin care related to the aging \\ process: integrative review
}

Análisis del efecto de la radiofrequencia em el tratamiento de flacidez cutánea relacionada al proceso de envejecimiento: revisión integrativa

Eduardo Kellyton de Oliveira Costa ${ }^{1 *}$, Daniela Lima de Almeida1, Francisca Taysa de Abreu Silva ${ }^{1}$, Fernanda Maria dos Reis Borges ${ }^{1}$,Consoello Vieira Pedrosa ${ }^{1}$, Edilane Rodrigues de Alencar ${ }^{1}$, Ana Oglády Jansen Pereira da Silveira Lacerda ${ }^{1}$, Naynne Fernanda Galvão de Oliveira ${ }^{1}$, Diego Rodrigues Pessoa ${ }^{1}$.

\section{RESUMO}

Objetivo: Este trabalho tem como objetivo realizar revisão integrativa para avaliar o efeito da radiofrequência no tratamento e prevenção de flacidez cutânea relacionada ao processo de envelhecimento. Métodos: $O$ estudo baseou-se na investigação de artigos publicados nas bases de dados PubMed/MedLine, LiLacs e SciELo, entre janeiro de 2012 novembro de 2019, combinando os descritores "flacidez cutânea", "radiofrequência", "envelhecimento" e "efeito" aos descritores booleanos nas mencionadas fontes de busca. Vinte e nove artigos foram encontrados neste estudo; em seguida aplicaram-se os critérios de inclusão e exclusão, e apenas 7 foram incluídos. Resultados: De acordo com os resultados dos artigos encontrados, há uma abordagem da Radiofrequência no tratamento da flacidez cutânea relacionada ao processo de envelhecimento, em que se observou redução significativa da flacidez cutânea, assim como das rugas, linhas de expressão e aspecto da pele. Conclusão: A Radiofrequência no tratamento da flacidez cutânea relacionada ao processo de envelhecimento mostrou-se eficiente.

Palavras-Chaves: Ondas de Radiofrequência, Tratamento, Envelhecimento.

\begin{abstract}
Objective: This work aims to perform an integrative review to evaluate the effect of radiofrequency in the treatment and prevention of cutaneous flaccidity related to the aging process. Methods: The study was based on the investigation of articles published in the PubMed / MedLine, LiLacs and SciELo databases between January 2012 and November 2019, combining the descriptors "skin flaccidity", "radiofrequency", "aging" and "effect "To the Boolean descriptors in the afore mentioned search sources. Twenty-nine articles were found in this study; then the inclusion and exclusion criteria were applied, and only 7 were included. Results: According to the results of the articles found, there is a Radiofrequency approach in the treatment of cutaneous flaccidity related to the aging process, in which a significant reduction of skin flaccidity was observed, as well as wrinkles, expression lines and skin appearance. Conclusion: Radiofrequency in the treatment of cutaneous flaccidity related to the aging process was efficient.
\end{abstract}

Key Words: Radiofrequency Waves, Treatment, Aging.

${ }^{1}$ Centro Universitário Santo Agostinho (UNIFSA), Teresina- PI. *E-mail: eduardor7k@gmail.com

SUBMETIDO EM: 4/2019

ACEITO EM: 5/2019

PUBLICADO EM: 8/2019 


\section{RESUMEN}

Objetivo: Este trabajo tiene como objetivo realizar una revisión integrativa para evaluar el efecto de la radiofrecuencia en el tratamiento y prevención de flacidez cutánea relacionada al proceso de envejecimiento. Métodos: El estudio se basó en la investigación de artículos publicados en las bases de datos PubMed / MedLine, LiLacs y SciELo, entre enero de 2012 de noviembre de 2019, combinando los descriptores "flacidez cutánea", "radiofrecuencia", "envejecimiento" y "efecto "A los descriptores booleanos en las mencionadas fuentes de búsqueda. Veintiséis artículos fueron encontrados en este estudio; a continuación se aplicaron los criterios de inclusión y exclusión, y sólo se incluyeron 7. Resultados: De acuerdo con los resultados de los artículos encontrados, hay un abordaje de la radiofrecuencia en el tratamiento de la flacidez cutánea relacionada al proceso de envejecimiento, en que se observó una reducción significativa de la flacidez cutánea, así como de las arrugas, líneas de expresión y aspecto de la piel . Conclusión: La radiofrecuencia en el tratamiento de la flacidez cutánea relacionada con el proceso de envejecimiento se mostró eficiente.

Palabras clave: Ondas de Radiofrecuencia, Tratamiento, Envejecimiento.

\section{INTRODUÇÃO}

O envelhecimento pode ser definido como sendo um conjunto de alterações morfológicas, fisiológicas e bioquímicas inevitáveis que ocorrem no organismo ao longo de nossas vidas. As modificações protéicas e a proliferação celular diminuem, ocorre a alteração do material genético por meio de enzimas decorrente do envelhecimento cronológico cutâneo (FACCHINETTI JB, DE SOUZA JS, SANTOS KTP, 2017).

Com o envelhecimento, a capacidade de regular as comutações aquosas e a proliferação celular dos tecidos ficam menos eficiente, com perda da elasticidade, resistência e tônus dos tecidos. Oxidações enzimáticas e química envolto ao desenvolvimento de radicais livres (RL) antecipam esse processo de enfraquecimento celular a nível tecidual (HIRATA LL, SATO MEO, SANTOS CAM,2004).

A principal característica da flacidez cutânea ocorre quando o colágeno se torna gradualmente mais rígido e a elastina vai perdendo suas propriedades físicas. A flacidez é uma nomenclatura que menciona à atributo ou situação de frouxidão tecidual. Surge comumente a partir da terceira década de vida da mulher, o que é bem notório no corpo sendo visível principalmente na região abdômen, coxas, glúteos, rosto e braços. Determinada pela existência desorganizada e diminuição das atividades dos fibroblastos, o que ocasionam uma redução na proliferação de colágeno. Levando a formação da flacidez cutânea estando relacionada diretamente com a atividade do tecido conjuntivo de manutenção (TESTO NAP, NARDINO D, PIVATO L, 2010).

Silva RMV, et al. (2017) afirma que a busca pelo rejuvenescimento nos dias atuais é uma preocupação comum. Atualmente, a literatura vigente aponta que existem inúmeros tratamentos para rejuvenescimento facial, dentre os métodos mais utilizados, destaca-se a Radiofrequência (RF). Os tratamentos de Radiofrequência são tradicionalmente titulados como não-ablativos e são ideais para aqueles que desejam evitar o tempo de recuperação estética (BEASLEY KL, WEISS RA, 2014; SADICK N, ROTHAUS KO, 2016).

A Radiofrequência é utilizada no tratamento de flacidez cutânea, sendo um dos maiores problemas causados pelo envelhecimento, a qual atua na derme, modelando fibras de colágeno e suavizando rugas da face, esse artifício gera o recondicionamento da pele aperfeiçoando a elasticidade e a eficácia tensora dos tecidos miscigenados por colágeno, com fabricação de novas fibras de qualidade determinando uma melhora da flacidez, tanto facial como corporal. Os efeitos fisiológicos da radiofrequência no combate à flacidez são vasodilatação e aumento da circulação sanguínea, além da elevação da temperatura que produz vasodilatação local, há também estímulo do aporte de nutrientes e oxigênio, acelerando a eliminação dos catabólitos (MACIEL D, OLIVEIRA GG, 2011).

Diante do exposto, este trabalho tem como objetivo realizar uma revisão integrativa através da leitura de artigos nacionais e internacionais que avaliem o efeito da radiofrequência no tratamento e prevenção de flacidez cutânea relacionada ao processo de envelhecimento. 


\section{MÉTODOS}

Esta revisão integrativa investigou estudos publicados em bases de dados eletrônicas no período entre janeiro de 2012 e fevereiro de 2019, utilizando artigos que abordaram o efeito da radiofrequência no tratamento e prevenção de flacidez cutânea relacionada ao processo do envelhecimento. Para isso, foram consultadas as bases de dados PubMed/MedLine, LiLacs e SCleLo.

Os termos utilizados para a busca dos estudos foram "radiofrequency", "Aging ", Saggingskin" e "effect", assim como "Flacidez cutânea"; "Radiofrequência", "envelhecimento" e "efeito", em diferentes combinações, nas referidas bibliotecas de busca e associadas aos descritores booleanos. A pesquisa foi realizada no período de fevereiro a abril de 2019, sendo selecionados artigos de ensaios clínicos, controlados, randomizados, comparativo, e estudos de coorte. Para tanto utilizaram-se os idiomas português e inglês, e foram escolhidos trabalhos publicados nos últimos 8 anos (2012 a 2019).

Os artigos foram ponderados por 7 pesquisadores: primeiramente foram feitas as apreciações do título e, em seguida, a leitura crítica do resumo para aproveitamento dos discernimentos de exclusão. Posteriormente a triagem dos artigos segundo os critérios de inclusão, foi desempenhada a leitura de todos eles, dos quais se extraíram os subsídios de interesse, como também foram averiguadas suas listas de referências, visando localizar artigos suplementares para a edificação da revisão.

Foram excluídos teses e dissertações, artigos de revisão literatura e sistemática, assim como artigos cujo texto completo não fosse disponível nem mesmo depois da busca em biblioteca de referência, ou seja, aqueles cuja obtenção, por algumas dificuldades técnicas, não fosse possível. Com os artigos escolhidos, foram tabuladas as centrais variáveis de instância, como a dimensão da amostra, o objetivo, a metodologia, os parâmetros analisados e os fundamentais resultados.

\section{RESULTADOS E DISCUSSÃO}

A flacidez cutânea ocorre devido à formação de radicais livres que em conjunto a uma série de alterações devido ao envelhecimento natural da pele faz com que haja a necessidade da utilização da tecnologia para promover o tratamento da mesma, desse modo a aplicação da radiofrequência tornou-se uma forte aliada para diminuir os efeitos das rugas e da flacidez na pele (CARVALHO GF, SILVA RMV, MESQUITA FILHO JJ. et al. 2011). Diante disso, diversos autores realizam estudos para se ter um protocolo que possa atender melhor essas necessidades.

Busnardo VL, Azevedo MF (2012) por exemplo, afirmaram que a radiofrequência é uma técnica diligente quando bem preconizado, para atenuação e terapia das rugas e flacidez da face. Para aproveitamento deste é imprescindível o conhecimento dos parâmetros eletrotermofototerapia e físicos, com habilidades para manejo do cabeçote e domínio de temperatura para não ocasionar efeitos adversos.

Nienkoett ER et al. (2012) avaliaram a caracterização da pele, como: textura, hidratação, flacidez, coloração e a presença de manchas, logo após o protocolo de Radiofrequência por 30 minutos, realizado oito sessões, uma vez por semana. Pode-se verificar a melhora da flacidez facial, textura, coloração e atenuação das rugas superficiais, assim como, a diminuição de linhas de expressão e no aspecto geral da pele de todas as pacientes.

Lima EA, et al. (2015) estabeleceram que a radiofrequência é uma proposta terapêutica promissora para o rejuvenescimento periorbital, principalmente quando não há indicação ou desejo de cirurgia convencional e quando a pele fina, flácida e enrugada é a queixa mais marcante. E que o retorno às atividades é rápido e os efeitos adversos foram poucos observados no grupo avaliado.

Primeiramente, foram localizados 29 artigos. Desses, 16 eram duplicados nas referidas bases de dados, restando 13 artigos para a leitura dos títulos, dos quais dois foram eliminados por não equivaler as adequações dos critérios apontados. 11 artigos elegidos para análise dos resumos, quatro foram excluídos, restando apenas 7 para tomar parte deste estudo. Posteriormente a revisão das referências destes, nenhum 
outro trabalho foi incorporado. Ao fim das investigações dos dados, a revisão foi miscigenada por sete artigos, e o fluxograma da Figura 1 detalha todos os artifícios de busca pelas pesquisas elegidas para a confecção desta revisão.

Figura 1 - Fluxograma dos estudos identificados.
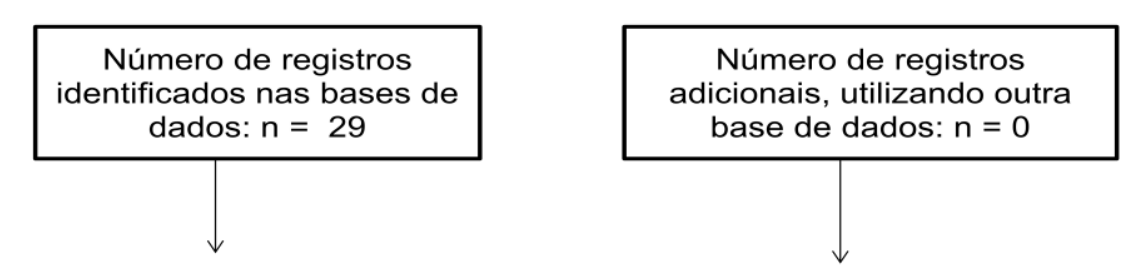

Número de artigos excluídos após apresentarem duplicidade nas bases de dados de pesquisas: $n=16$

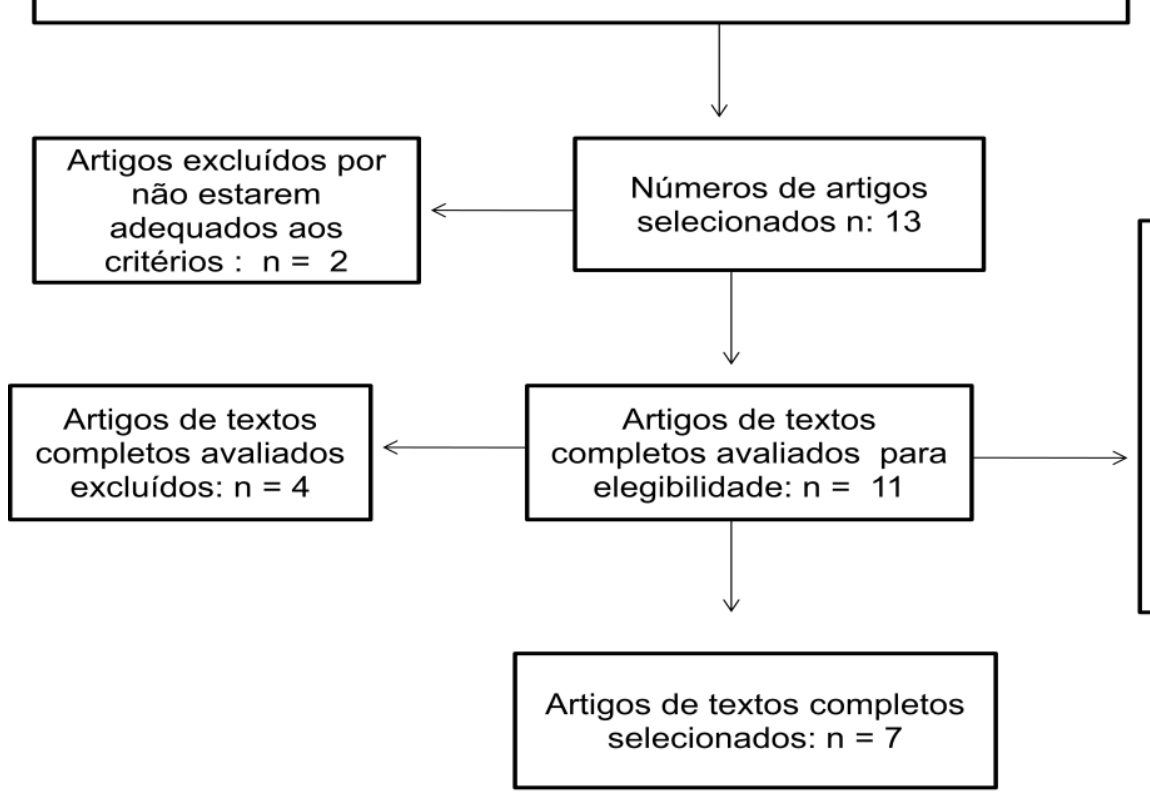

BUSNARDO VL, AZEVEDO MF(2012).

NIENKOETTER, et al. (2013).

LIMA, et al. (2015).

MARCHI JP, et al. (2016).

WAKADE DV, et al. (2015).

FACCHINETTI, et al. (2017).

SILVA RMW, et al. (2017).

Fonte: Elaborado pelos autores, 2019.

Marchi JP (2016) demonstraram efeitos significativos na redução da extensão e profundidade das rugas e na melhora do quadro de flacidez cutânea, além de melhora visível no aspecto da pele, ou seja, é um tratamento completo para revitalização cutânea.

Wakade DV, et al. (2015) realizaram um estudo comparando a eficácia da radiofrequência monolar e ácido glicolítico no rejuvenescimento fácil, foi verificado que a radiofrequência monopolar teve melhor resultado, não foi significativo. Facchinetti JB, et al. (2017) indicaram que o tratamento com base na Radiofrequência se mostrou positivo ao verificar uma redução da área das rugas e flacidez cutânea que foram submetidas ao tratamento. Apesar da melhora geral do aspecto, alguns pacientes demonstraram uma mudança mais evidente do que outras. Logo, é importante ressaltar que diversos fatores são responsáveis pelo aparecimento das rugas e consequentemente, poderão influenciar nas respostas ao tratamento como, por exemplo, alimentação, pratica de atividade física e tabagismo. 
Os estudos tiveram suas informações resumidas nos tópicos: autor/ano, objetivo, amostra, metodologia e resultados, os quais foram tabulados e apresentados na Tabela 1.

Tabela 1 - Delineamento, métodos e principais desfechos dos estudos selecionados.

\begin{tabular}{|c|c|c|c|c|}
\hline Autor/ano & Objetivo & Amostra & Metodologia & Resultados \\
\hline $\begin{array}{ll}\text { BUSNARDO } & \text { VL, } \\
\text { AZEVEDO } & \text { MF } \\
(2012) & \end{array}$ & $\begin{array}{l}\text { Analisar os efeitos de um } \\
\text { programa de tratamento de } 8 \\
\text { semanas de RF na melhora das } \\
\text { rugas, da tonicidade, do aspecto } \\
\text { da pele e do contorno facial. }\end{array}$ & $\begin{array}{c}16 \\
\text { Voluntárias }\end{array}$ & $\begin{array}{lr}\text { As voluntárias foram } \\
\text { divididas em } 2 \\
\text { grupos: } \\
\text { Grupo 1 (controle) } \\
\text { Grupo } \\
\text { (experimental) } & -8 \\
\text { semanas } & \text { de } \\
\text { tratamento com a } \\
\text { RF. }\end{array}$ & $\begin{array}{l}\text { Redução das rugas } \\
\text { Melhora da flacidez. }\end{array}$ \\
\hline $\begin{array}{l}\text { NIENKOETT ER, } \\
\text { et al. (2013) }\end{array}$ & $\begin{array}{l}\text { O objetivo deste estudo foi } \\
\text { verificar os efeitos do uso da } \\
\text { radiofrequência bipolar marca } \\
\text { CECBRA® no tratamento de } \\
\text { flacidez facial. }\end{array}$ & $\begin{array}{c}10 \\
\text { Voluntárias }\end{array}$ & $\begin{array}{l}\text { As voluntárias } \\
\text { foram submetidas a } \\
\text { tratamento facial por } \\
\text { meio da RF, } 1 \text { vez } \\
\text { por semana }\end{array}$ & $\begin{array}{l}\text { Melhora da flacidez; } \\
\text { Redução das linhas de } \\
\text { expressão facial } \\
\text { Melhora da coloração } \\
\text { e textura } \\
\text { Redução das rugas }\end{array}$ \\
\hline $\begin{array}{l}\text { LIMA EA, et al. } \\
\text { (2015) }\end{array}$ & $\begin{array}{l}\text { Avaliar a eficácia da } \\
\text { radiofrequência por micro } \\
\text { agulhas no rejuvenescimento da } \\
\text { região periorbitária. }\end{array}$ & $\begin{array}{c}19 \\
\text { Voluntárias }\end{array}$ & $\begin{array}{l}\text { Os participantes } \\
\text { foram submetidos a } \\
\text { tratamento } \\
\text { contínuos pela RF } \\
\text { em modo pulsado } \\
\text { (especificar o tempo } \\
\text { experimental) }\end{array}$ & $\begin{array}{l}\text { Melhora da flacidez; } \\
\text { Redução das linhas de } \\
\text { expressão facial } \\
\text { Melhora da coloração } \\
\text { e textura da pele } \\
\text { Redução das rugas }\end{array}$ \\
\hline $\begin{array}{l}\text { MARCHI JP, et } \\
\text { al. (2016) }\end{array}$ & $\begin{array}{l}\text { O estudo teve como objetivo } \\
\text { avaliar os efeitos do tratamento } \\
\text { facial com radiofrequência em } \\
\text { pele de voluntárias tabagistas e } \\
\text { não-tabagistas }\end{array}$ & $\begin{array}{c}8 \\
\text { Voluntárias }\end{array}$ & $\begin{array}{l}\text { As participantes } \\
\text { foram submetidas a } \\
\text { tratamento com a RF } \\
\text { por } 10 \text { sessões, com } \\
\text { duração de } 60 \\
\text { minutos. }\end{array}$ & $\begin{array}{lr}\text { Diminuição } & \text { da } \\
\text { extensão } & \text { e } \\
\text { profundidade das } \\
\text { rugas } \\
\text { Minimização } \\
\text { flacidez cutânea } \\
\text { Aumento nos níveis de } \\
\text { hidratação e nutrição } \\
\text { tecidual melhorando o } \\
\text { aspecto cutâneo } \\
\begin{array}{l}\text { Melhora do } \\
\text { clareamento facial }\end{array}\end{array}$ \\
\hline $\begin{array}{l}\text { WAKADE DV, et } \\
\text { al. (2015) }\end{array}$ & $\begin{array}{l}\text { Comparar os benefícios da } \\
\text { radiofrequência e ácido glicólico } \\
\text { descasca em rejuvenescimento } \\
\text { facial com relação a a } \\
\text { histopatologia e Ultra } \\
\text { biomicroscopicecografia (UBM). }\end{array}$ & $\begin{array}{c}40 \\
\text { Voluntárias }\end{array}$ & $\begin{array}{l}\text { As voluntárias foram } \\
\text { submetidas há } 4 \\
\text { sessões r de } \\
\text { tratamento por } \\
\text { semana ao longo de } \\
3 \text { semanas. }\end{array}$ & $\begin{array}{l}\text { Melhora do } \\
\text { rejuvenescimento } \\
\text { facial. }\end{array}$ \\
\hline $\begin{array}{l}\text { FACCHINETTI } \\
\text { JB, et al. (2017) }\end{array}$ & $\begin{array}{l}\text { Avaliar a eficácia da } \\
\text { radiofrequência } \\
\text { rejuvenescimento facial. }\end{array}$ & $\begin{array}{c}8 \\
\text { Voluntárias }\end{array}$ & $\begin{array}{l}\text { As voluntárias foram } \\
\text { submetidas a } 10 \\
\text { sessões de } \\
\text { região nacial. }\end{array}$ & $\begin{array}{l}\text { Melhora da flacidez; } \\
\text { Redução das linhas de } \\
\text { expressão facial } \\
\text { Melhora da coloração } \\
\text { e textura da pele } \\
\text { Redução das rugas }\end{array}$ \\
\hline $\begin{array}{l}\text { SILVA RMW, et } \\
\text { al. (2017) }\end{array}$ & $\begin{array}{l}\text { Investigar os efeitos } \\
\text { radiofrequência } \\
\text { rejuvenescimento facial. }\end{array}$ & $\begin{array}{c}40 \\
\text { Voluntárias }\end{array}$ & 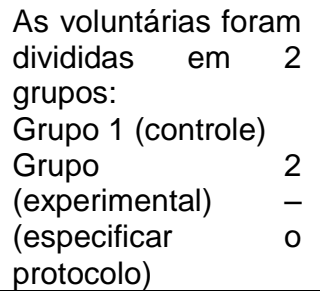 & $\begin{array}{l}\text { Melhora da flacidez; } \\
\text { Redução das linhas de } \\
\text { expressão facial }\end{array}$ \\
\hline
\end{tabular}

Fonte: Elaborado pelos autores, 2019. 
Silva RMW, et al. (2017) afirma que a radiofrequência não promoveu alterações estatísticas significativas no tamanho e angulação do sulco nasogeniano de mulheres com flacidez facial, no entanto na análise por profissionais especialista verificou-se uma melhora clínica maior no grupo que realizou a aplicação da radiofrequência.

\section{CONSIDERAÇÕES FINAIS}

A radiofrequência permite a correção de sinais de envelhecimento, a partir de um campo eletromagnético que induz o movimento de partículas ionizadas, promovendo o aquecimento tecidual por diatermia. Desse modo o artigo obteve significativos resultados com relação ao proposto, no entanto se faz necessário a realização de mais estudos abrangentes relacionados a radiofrequência como método de tratamento para a flacidez tissular.

\section{REFERÊNCIAS}

1. BEASLEY KL, WEISS RA. Radiofrequency in cosmetic dermatology. Dermatologic clinics. 2014; 32(1): 79-90.

2. BUSNARDO VL, AZEVEDO MF. Estudo dos efeitos da radiofrequência no tratamento facial em mulheres entre $50 \mathrm{e}$ 60 anos de idade, 2012.

3. DE CARVALHO, GF, SILVA, RMV, MESQUITA FILHO, JJ. et al. Avaliação dos efeitos da radiofrequência no tecido conjuntivo. Revista brasileira de medicina, v. 68, p. 10-25, 2011.

4. FACCHINETTI JB, DE SOUZA JS, SANTOS KTP. Radiofrequência no Rejuvenescimento Facial. Id Online Revista de Psicologia. 2017; 11(38): 336-348.

5. HIRATA LL, SATO MEO, SANTOS CAM. Radicais livres e o envelheci mento cutâneo. Acta Farm. Bonaerense. 2004; 23(3): 418-424.

6. KEDE, MPV, SABATOVICH O. Dermatologia estética. in: Dermatologia estética. 2009.

7. LIMA EA, RECIFE CMR, LIMA EA. Radiofrequência pulsada com multi agulhas: uma proposta terapêutica em rugas, flacidez e pigmentação periorbital. Surgical \& Cosmetic Dermatology. 2015; 7(3): 223-226.

8. MACIEL D, OLIVEIRA GG. Prevenção do envelhecimento cutâneo e atenuação de linhas de expressão pelo aumento da síntese de colágeno." Multiprofissional em Saúde: Atenção ao Idoso. 2011.

9. MARCHI JP. Efetividade da radiofrequência no tratamento facial de voluntárias tabagistas e não tabagistas. Arquivos de Ciências da Saúde da UNIPAR. 2016; 20(2):1-8.

10. NIENKOETTE RL, HELLMANN LT, GONÇALVES VP. Efeitos da radiofrequência no tratamento de flacidez facial em mulheres. Revistas Eletrônicas de Estética e Cosmética, Florianópolis. 2012; 3:1-8.

11. NORONHA L. "Estudo morfométrico e morfológico da cicatrização após uso do laser erbium: YAG em tecidos cutâneos de ratos. J BrasPatol Med. 2004; 40(1): 41-48.

12. SADICK N, ROTHAUS KO. Aesthetic applications of radio frequency devices. Clinics in plastic surgery. 2016; 43(3): 557-565.

13. SILVA RMV. Efeitos da radiofrequência no rejuvenescimento facial: estudo experimental. ConScientiae Saúde. 2017; 16(2): 194-200.

14. TESTO NAP, NARDINO D, PIVATO L. Envelhecimento cutâneo: teoria dos radicais livres e tratamentos visando a prevenção e o rejuvenescimento. Revista Uningá Review. 2010; 1(1): 1-8.

15. WAKADEDV,NAYAK CS,BHATT KD. A study comparing the efficacy of monopolar radiofrequency and glycolicacidpeels in facial rejuvenation of aging skin using histopathology and ultra-biomicroscopic sonography (UBM)-na evidence-based study. Acta Medica (HradecKralove). 2016; 59(1): 14-17. 